\title{
A Guitaring Technique with 035 Wire and Perforated Coronary Balloon for Thrombolysis in the Treatment of Acute Deep Vein Thrombosis
}

\author{
Mark Christopher Arokiaraj \\ Cardiology, Pondicherry Institute of Medical Sciences, Pondicherry, India
}

\begin{abstract}
A patient who underwent thrombolysis and inferior vena cava filter implantation for acute deep vein thrombosis treatment nine years before, presented with deep vein thrombosis on the other limb. The venous angiogram showed deep vein thrombosis in the ilio-femoral vessels. Through left femoral vein puncture, a $6 \mathrm{~F}$ right Judkins diagnostic catheter was advanced up to the proximal iliac veins, and further advancement was not possible. Hence, a 035 hydrophilic wire was advanced, and through a guitaring technique, the clots were disrupted at the mid-thigh level. Through a 014 coronary wire, a punctured coronary balloon was placed at the distal mid-thigh level, and intravascular thrombolysis was performed for 12 hours, followed by removal of the balloon and the right coronary Judkins catheters. The leg swelling reduced in the first day, and subsequently, the patient was followed up for 7 months with anticoagulation and good resolution of the symptoms.
\end{abstract}

Keywords: deep vein thrombosis, punctured coronary balloon, guitaring technique, local thrombolysis

\section{ARTICLE HISTORY}

Received: June 30, 2019

Accepted: September 12, 2019

\section{CORRESPONDENCE}

Mark Christopher Arokiaraj

Pondicherry Institute of Medical Sciences Kalathumettupathai,

Ganapathichettikulam, Village No.20, Kalapet, Puducherry, 605014, India

Tel: +919751783843

E-mail: christomark@gmail.com

\section{INTRODUCTION}

Deep venous thromboembolism is common in clinical practice. The incidence of this condition is about 100 in 100,000 patient-years. ${ }^{1}$ Pulmonary embolism is common in patients with deep venous thrombosis. ${ }^{2}$ In about $45 \%$ of patients with pulmonary embolism, associated deep venous thrombosis is present. ${ }^{3}$ Post-thrombotic syndrome, a debilitating associated condition, is common in patients with extensive deep vein thrombosis with an incidence of 20 to $50 \%$ in various studies, especially in diabetics. ${ }^{4}$ In this case report, we describe a novel technique used for the disruption of clots in the deep vein thrombosis segment.

\section{CASE REPORT}

A 40-year-old woman presented with swelling in the right leg for two days. She had a history of deep vein thrombo- sis of the left lower limb nine years before, for which she underwent peripheral balloon-directed thrombolysis and inferior vena cava (IVC) filter placement.

Work-up for primary coagulation abnormalities during the first episode was unremarkable. Also, immunologic assessment for autoimmune disorders did not reveal any abnormalities in the past. The patient was prescribed warfarin and clopidogrel $75 \mathrm{mg}$ once a day, for which she was not regularly compliant.

She had also a known perennial asthma, for which she was under treatment with inhalator steroids, and she received therapy for exacerbations of asthma during the winter season. At the same time, she was diagnosed with bilateral allergic nasal polyps for which she has been advised for surgery, being scheduled for operation in the near future. Her antinuclear antibody (ANA), rheumatoid arthritis (RA) factor, anti-neutrophil cytoplasmic antibody (ANCA), and antiphospholipid antibody tests were not reactive. 

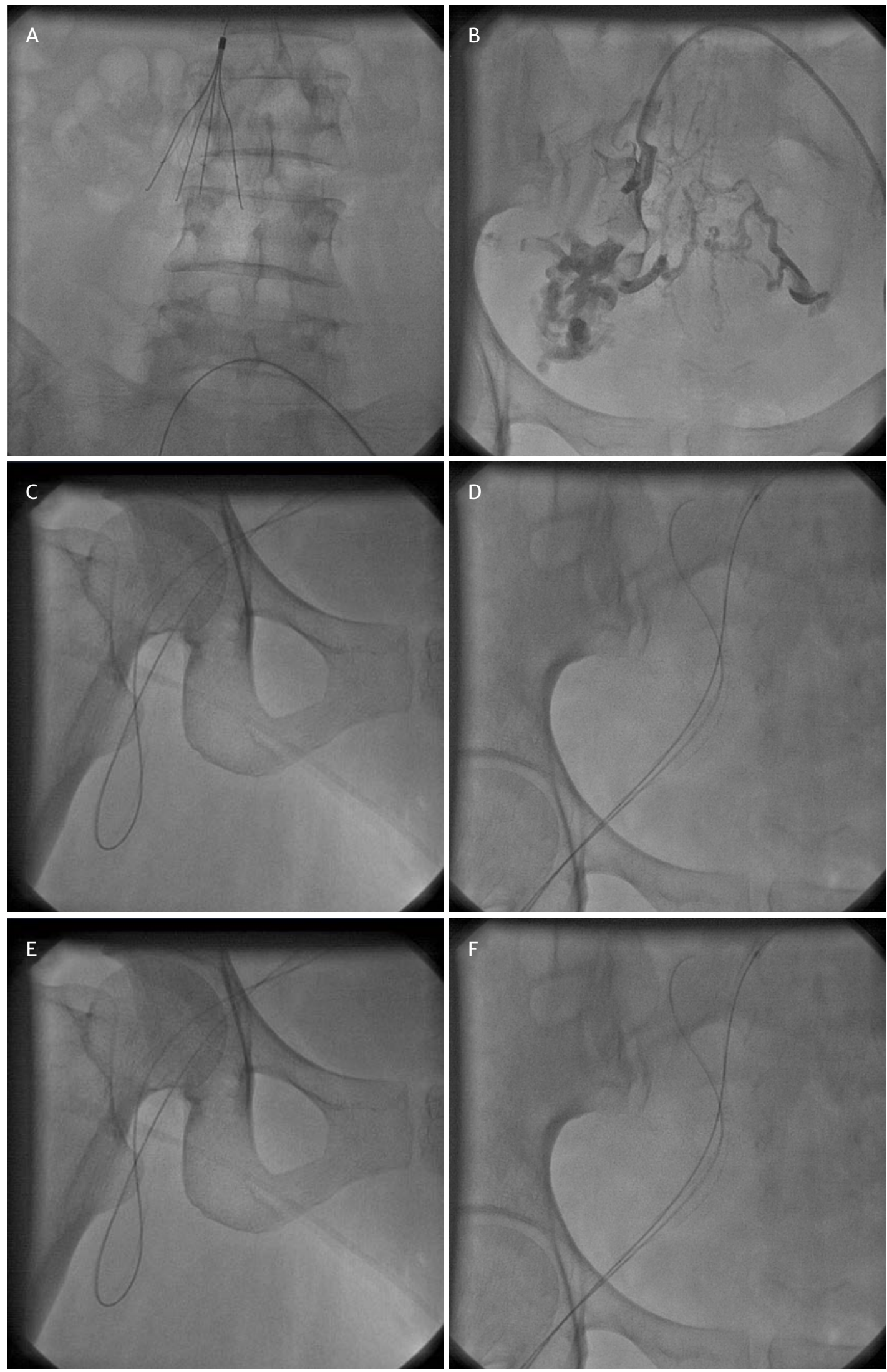

F

FIGURE 1. A - The IVC filter placed in the past; B - Venous angiogram in the proximal iliac veins through the $6 \mathrm{~F}$ diagnostic catheter; C-E - The advancement of the 034 hydrophilic wire; $\mathbf{F}$ - The placement of perforated coronary balloon (arrow) at the mid-thigh level for thrombolysis 


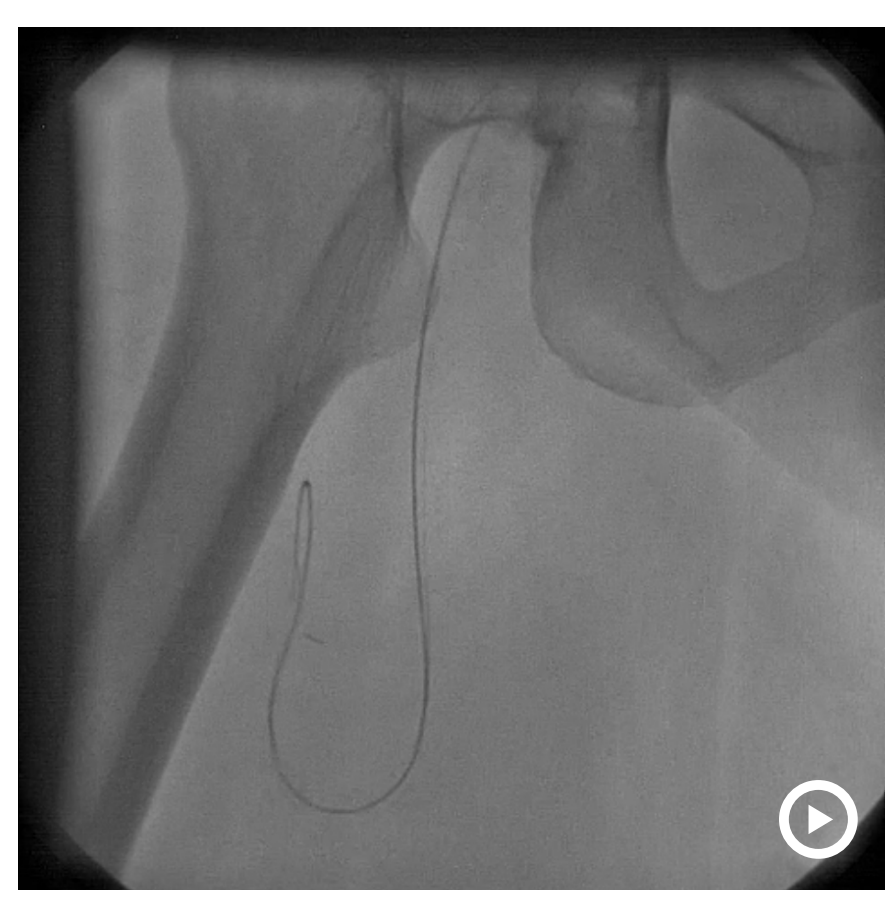

MOVING IMAGE 1. Guitaring technique with 035 wire

This episode of deep vein thrombosis in the right leg showed clots in the proximal iliac veins extending to the popliteal veins and below on Doppler ultrasound. She had significant swelling extending from the right groin, thighs, and sacral region, extending to the entire right lower limb. She was started on enoxaparin and antiplatelets.

Since her symptoms were significant, thrombolysis and venous balloon angioplasty were planned to the right leg. Initial fluoroscopy showed the IVC filter (Gunter Tulip) inserted nine years before, in the same location (Figure 1 panel A). Through a left femoral approach, a right Judkins $6 \mathrm{~F}$ diagnostic catheter was placed in the proximal common iliac vein (Figure 1 panel B), and 035 Terumo wire was advanced to the mid-thigh level (Figure 1 panels $\mathrm{C}-\mathrm{E}$ ). Further advancement of the right Judkins diagnostic catheter was not possible due to clots in proximal common iliac vein. Repeated crossing of the lesion was attempted using $5 \mathrm{~F}$ Judkins right diagnostic catheter, $6 \mathrm{~F}$ guiding catheter, and $6 \mathrm{~F}$ multipurpose catheter, however this remained unsuccessful. This was due to old hardened thrombus in the common iliac vein bifurcation as a sequelae of the deep vein thrombosis nine years earlier. Hence, only the hydrophilic 035 wire (Terumo) was advanced to the mid-femoral level, and mechanical disruption of the clot was performed with the 035 wire. The 035 wire was moved in a fast sequence similar to guitaring for about 30 to $45 \mathrm{~s}$ (video).

The process of guitaring is a simple, rapid movement of the 035 wire sideways, and to-and-from combination movements in a fast and rotatory manner after placement of the wire inside the venous segment with clot. A peripheral balloon was then advanced with an attempt to perform venous balloon angioplasty of the right iliofemoral veins. However, the peripheral balloon could not cross the proximal right iliac veins. Hence, a 014 balanced middleweight wire (BMW) was advanced up to a mid-thigh level with the support of a $2.5 \times 12 \mathrm{~mm}$ coronary balloon (Sprinter), which was inflated outside and punctured with a needle. The balloon catheter was advanced through a 014 wire and was placed at the level of the mid-femoral vein (Figure 1 panel F). Aspirin and clopidogrel were given at doses of $150 \mathrm{mg}$ each. Streptokinase was used for thrombolysis and was administered at a dose of $5 \mathrm{~mL} / \mathrm{h}$ at a concentration of 15,000 $\mathrm{IU} / \mathrm{mL}$. The infusion was given for about 12 hours. The patient developed shivering and fever during the infusion process, for which antibiotics and paracetamol injections were given. After thrombolysis, the balloon and the $6 \mathrm{~F}$ right Judkins diagnostic catheter placed in the proximal right iliac vein were removed. Subsequently, the patient was again started on heparin, followed by warfarin and clopidogrel.

Within 12 hours of the procedure, the subcutaneous tissue in the right thigh and right leg softened, and the swelling reduced within three days. Clopidogrel was stopped after 1 month.

Consent for publication of this case was obtained, and all the related procedures were performed in accordance with the declaration of Helsinki.

The patient was followed up for 7 months. The limb swelling decreased significantly, and the patient is currently on oral anticoagulation with warfarin. In this case, mechanical disruption of the clots in the iliofemoral veins was performed with the 035 Terumo wire itself. Also, the coronary balloon was successfully used for thrombolysis.

\section{DISCUSSIONS}

This is the first report of a new method for treating iliofemoral deep vein thrombosis that uses a coronary balloon for local administration of thrombolysis and a 035 Terumo wire for disruption of the clots. This technique is simple, the necessary devices are available in all cardiac catheterization laboratories, and the method can be recommended for all patients with deep vein thrombosis after placement of an IVC filter. The technique was performed in a single case, and further evaluation is required for its validation. In a previous study conducted in our center, peripheral balloons were used for thrombolysis and venous angioplasty. ${ }^{5}$ In this case, the IVC filter had been placed nine years before, the current clot formation was at the level of the iliofemoral veins, and the IVC filter was free of clots. This shows that 
the IVC filter inserted in the past was not associated with the current thrombosis. The use of coronary balloon and wires is a minimally invasive procedure, and thereby would reduce the potential risk of dislodgement of small clots from the deep vein thrombus, which could escape the IVC filter and embolize upwards in the inferior vena cava, even though large clots are usually trapped by the filter. The currently available techniques involve the use of thrombus aspiration catheters, catheter-directed thrombolysis, or angio-jet techniques. ${ }^{6,7}$ Thrombus aspiration is particularly useful for very soft clots. However, in the majority of cases, the clots are not very soft, which limits the potential application of this technique. Catheter-directed thrombolysis is feasible, but in many cases catheter advancement is restricted in the proximal areas. Angio-jet is usually efficient, but has a limited availability in many centers and can cause hemolysis or dissections of the vascular wall.

\section{CONCLUSIONS}

Mechanical disruption of clots with a 035 wire can represent a therapeutic alternative in venous procedures, and thrombolysis performed via perforated coronary balloon is possible as a simple technique for acute deep vein thrombosis treatment after the placement of IVC filter. However, further studies are required to validate this novel technique.

\section{CONFLICT OF INTEREST}

Nothing to declare.

\section{REFERENCES}

1. Heit JA. Epidemiology of venous thromboembolism. Nat Rev Cardiol. 2015;12:464-474. doi:10.1038/nrcardio.2015.83.

2. Goldhaber S, Bounameaux H. Pulmonary embolism and deep vein thrombosis. Lancet. 2012;379:1835-1846. doi: 10.1016/ S0140-6736(11)61904-1.

3. Lee JS, Moon T, Kim TH, et al. Deep Vein Thrombosis in Patients with Pulmonary Embolism: Prevalance, clinical significance and outcome. Vasc Specialist Int. 2016;32:166174. doi:10.5758/vsi.2016.32.4.166.

4. Farrell JJ, Sutter C, Tavri S, Patel I. Incidence and interventions for post-thrombotic syndrome. Cardiovasc Diagn Ther. 2016;6:623-631. doi:10.21037/cdt.2016.11.22.

5. Arokiaraj MC, Guerrero L, Levine R, Palacios I. Simultaneous Thrombolysis and Venous Angioplasty in Acute Iliofemoral Thrombosis after IVC filter Insertion and Novel Balloon Catheter Models. J Clin Exp Cardiolog. 2013;4:240. doi:10.4172/2155-9880.1000240.

6. Pollack C. Advanced Management of Acute Iliofemoral Deep Venous Thrombosis: Emergency Department and Beyond. Annals of Emergency Medicine. 2011;57:590-599. doi: 10.1016/j.annemergmed.2010.11.031.

7. Streiff MB, Agnelli G, Connors JM, et al. Guidance for the treatment of deep vein thrombosis and pulmonary embolism. J Thromb Thrombolysis. 2016;41:32-67. doi: 10.1007/s11239015-1317-0. 